\title{
Power Flow Efficiency of Linear and Nonlinear Physical Systems
}

\author{
Roberto Zanasi, Davide Tebaldi
}

\begin{abstract}
In this paper, an analytical procedure to derive the efficiency of linear and nonlinear physical systems is presented. This procedure allows to compute the efficiency map both on the plane of the input power variables and on the plane of the output power variables. Additionally, the paper highlights the parameters to be adjusted in order to enlarge the highefficiency region of the system. The presented procedure can also be used in conjunction with a least square algorithm in order to estimate the unknown parameters of the considered physical system. The effectiveness of the procedure has been tested in Matlab/Simulink to estimate the parameters of an actual PMSM electric motor. The obtained results show a very good matching between the actual and the estimated efficiency maps.
\end{abstract}

\section{INTRODUCTION}

The concept of efficiency is much appreciated in the world of industry as it is intuitive and allows the user to determine the cost, in terms of dissipated power, related to the introduction of a specific physical element within the overall system. Industries typically use simplified methods to derive the average efficiency of a physical system, and usually the computed efficiency refers only to a particular operating point. In this paper, the problem of the power flow-based efficiency of a physical system is addressed as a general case, and the analysis is applied to both linear and nonlinear systems. The presented procedure shows the most efficient operating regions for the considered physical system, thus providing an insight of how the overall performance of the system can be improved. In the paper, the presented procedure is applied to three practical case studies: a DC electric motor, a mechanical gear transmission system and a Permanent Magnet Synchronous Motor (PMSM). The presented analysis can also be used to identify the unknown parameters of the considered system if its efficiency map on the output plane is given. This identification method has been applied in this paper to the PMSM case study. The same problem has been addressed, for example, in [1] and in [2]. In [1] the authors propose a technique to identify the motor parameters by trying to adaptively minimize the current vector error between the considered model and the actual system. In [2] the authors estimate the motor parameters by using a $2^{\text {nd }}$-order Extended Kalman Filter. In this paper, a new method which allows to identify the motor parameters by trying to minimize the mean squared error between the efficiency points on the actual and estimated efficiency maps is presented. Finally, the presented method has been used to

Roberto Zanasi and Davide Tebaldi are with the Department of Engineering "Enzo Ferrari", University of Modena and Reggio Emilia, Modena, Italy, e-mail: roberto.zanasi@unimore.it, davide.tebaldi@unimore.it estimate the unknown parameters of an industrial three-phase electric motor: the obtained good matching between the actual and estimated efficiency maps shows the effectiveness of the proposed method.

\section{EFFICIENCY OF LINEAR SYSTEMS}

Let us consider a linear dissipative system $\mathbf{H}(s)$ characterized by two power sections, see Fig. 1 . Let $\left(y_{1}, u_{1}\right)$ and $\left(y_{2}, u_{2}\right)$ denote the power variables of the input and output sections, and let us suppose the positive directions of the

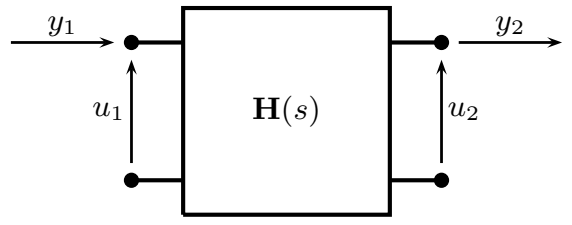

Fig. 1. Linear system $\mathbf{H}(s)$ characterized by two power sections

power variables to be chosen in such a way that the power flow $P_{1}=u_{1} y_{1}$ is positive if it is entering the system and power flow $P_{2}=u_{2} y_{2}$ is positive if it is exiting the system. The POG (standing for Power-Oriented Graphs, see [3]) state space equations of the considered linear system are:

$$
\left\{\begin{array}{r}
\mathbf{L} \dot{\mathbf{x}}=\mathbf{A} \mathbf{x}+\mathbf{B u} \\
\mathbf{y}=\mathbf{C x}+\mathbf{D u}
\end{array}, \quad \mathbf{u}=\left[\begin{array}{l}
u_{1} \\
u_{2}
\end{array}\right], \quad \mathbf{y}=\left[\begin{array}{l}
y_{1} \\
y_{2}
\end{array}\right] .\right.
$$

The transfer matrix $\mathbf{H}(s)$ can be determined as follows:

$$
\mathbf{H}(s)=\mathbf{C}(\mathbf{L} s-\mathbf{A})^{-1} \mathbf{B}+\mathbf{D} .
$$

In steady-state condition, matrix $\mathbf{H}(s)$ can be replaced with the static gain matrix $\mathbf{H}_{0}$ :

$$
\mathbf{H}_{0}=\left.\mathbf{H}(s)\right|_{s=0}=-\mathbf{C A}^{-1} \mathbf{B}+\mathbf{D} .
$$

Let vectors $\mathbf{y}, \mathbf{u}$ and matrix $\mathbf{H}_{0}$ be denoted as follows:

$$
\underbrace{\left[\begin{array}{l}
y_{1} \\
y_{2}
\end{array}\right]}_{\mathbf{y}}=\underbrace{\left[\begin{array}{cc}
a & b \\
c & -d
\end{array}\right]}_{\mathbf{H}_{0}} \underbrace{\left[\begin{array}{l}
u_{1} \\
u_{2}
\end{array}\right]}_{\mathbf{u}}
$$

When $\operatorname{det}\left(\mathbf{H}_{0}\right) \neq 0$, relation (2) can be graphically represented as shown in Fig. 2. System $\mathbf{H}(s)$ is dissipative if the total power flow $P_{i n}$ entering the system is positive:

$$
P_{\text {in }}=P_{1}-P_{2}=u_{1} y_{1}-u_{2} y_{2}=\left[u_{1}-u_{2}\right]\left[\begin{array}{l}
y_{1} \\
y_{2}
\end{array}\right]>0
$$

By using (2) and (3), power $P_{i n}$ can be expressed in the following way:

$$
P_{i n}=\left[\begin{array}{ll}
u_{1} & u_{2}
\end{array}\right] \underbrace{\left[\begin{array}{cc}
a & b \\
-c & d
\end{array}\right]}_{\mathbf{H}_{p}}\left[\begin{array}{l}
u_{1} \\
u_{2}
\end{array}\right]=\mathbf{u}^{\mathrm{T}} \mathbf{H}_{p} \mathbf{u}>0
$$




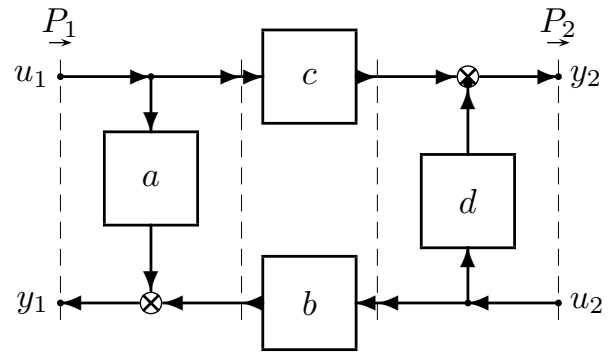

Fig. 2. Graphical representation of relation (2) when $\operatorname{det}\left(\mathbf{H}_{0}\right) \neq 0$.

One can easily prove that power $P_{i n}$ is positive if:

$$
a>0, \quad d>0, \quad \operatorname{det}\left(\mathbf{H}_{p}\right)=a d+b c>0 .
$$

When $\operatorname{det}\left(\mathbf{H}_{p}\right)=0$, power $P_{\text {in }}$ is equal to zero and the system exhibits internal steady-state conditions without dissipations.

\section{A. Definition of power flow efficiency}

Definition. The "power flow efficiency" $E(t)$ of a system having the structure shown in Fig. 1 is defined as follows:

$$
E(t)=\frac{u_{2}(t) y_{2}(t)}{u_{1}(t) y_{1}(t)}
$$

Relation (2) can also be expressed as follows:

$$
\left[\begin{array}{l}
u_{1} \\
y_{1}
\end{array}\right]=\left[\begin{array}{cc}
\frac{d}{c} & \frac{1}{c} \\
\frac{a d+b c}{c} & \frac{a}{c}
\end{array}\right]\left[\begin{array}{l}
u_{2} \\
y_{2}
\end{array}\right], \quad\left[\begin{array}{l}
u_{2} \\
y_{2}
\end{array}\right]=\left[\begin{array}{cc}
-\frac{a}{b} & \frac{1}{b} \\
\frac{a d+b c}{b} & -\frac{d}{b}
\end{array}\right]\left[\begin{array}{l}
u_{1} \\
y_{1}
\end{array}\right] .
$$

From (5) and (6), one can observe that the efficiency of the system only depends on coefficients $a, b, c, d$ which are present within the static gain matrix $\mathbf{H}_{0}$, see (2). From this observation, the following important property that applies to all physical systems can be derived.

Property 1: The efficiency of a physical system is not affected by the dynamic elements present within matrix $\mathbf{L}$.

By substituting (6) in (5), one obtains the following two equivalent ways of expressing efficiency $E(t)$ :

$$
\begin{gathered}
E\left(y_{2}, u_{2}\right)=\frac{c^{2} u_{2} y_{2}}{\left(d u_{2}+y_{2}\right)\left[(a d+b c) u_{2}+a y_{2}\right]} \\
E\left(y_{1}, u_{1}\right)=\frac{\left(-a u_{1}+y_{1}\right)\left[(a d+b c) u_{1}-d y_{1}\right]}{b^{2} u_{1} y_{1}}
\end{gathered}
$$

From (7) and (8), it follows that $E(t)$ can be given in two different equivalent ways: by plotting function $E\left(y_{2}, u_{2}\right)$ on the plane of the output variables $\left(y_{2}, u_{2}\right)$, or by plotting function $E\left(y_{1}, u_{1}\right)$ on the plane of the input variables $\left(y_{1}, u_{1}\right)$.

\section{B. Efficiency of linear systems on input and output planes.}

Property 2: The efficiency $E(t)$ of a linear system is constant along straight lines exiting from the origin of the output plane $\left(y_{2}, u_{2}\right)$ :

$$
u_{2}=\gamma y_{2}, \quad \gamma \in[0, \infty]
$$

and reaches its maximum value $E^{*}$ for:

$$
\gamma=\sqrt{\frac{a}{d(a d+c b)}}=\gamma^{*}
$$

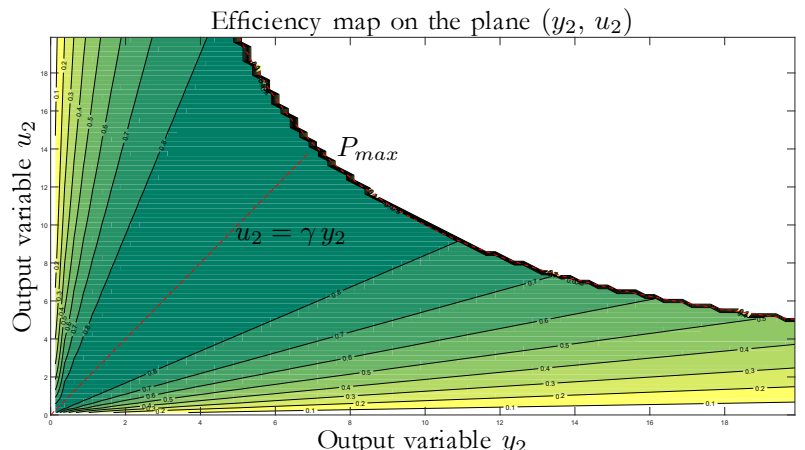

Fig. 3. Efficiency map shown on the plane of the output variables $\left(y_{2}, u_{2}\right)$.

Proof. By substituting (9) in (7), it is possible to express $E(t)$ as a function of parameter $\gamma$ :

$$
E(t)=\frac{c^{2} \gamma}{(d \gamma+1)[(a d+b c) \gamma+a]}=E(\gamma)
$$

Efficiency $E(\gamma)$ in (11) can also be rewritten as follows:

$$
E(\gamma)=\frac{c^{2} \gamma}{a+\beta \gamma+\delta \gamma^{2}} \text { where }\left\{\begin{array}{l}
\beta=2 a d+c b \\
\delta=d(a d+c b)
\end{array}\right.
$$

By deriving $E(\gamma)$ with respect to $\gamma$, one obtains:

$$
\frac{\partial E(\gamma)}{\partial \gamma}=\frac{c^{2}\left(a-\delta \gamma^{2}\right)}{\left(a+\beta \gamma+\delta \gamma^{2}\right)^{2}} .
$$

Efficiency $E(\gamma)$ is maximized when $\frac{\partial E(\gamma)}{\partial \gamma}=0$, that is when $\gamma=\sqrt{\frac{a}{\delta}}$. By using $\delta$ from (12), one directly obtains relation (10).

By means of (11), one obtains the maximum efficiency $E^{*}=$ $E\left(\gamma^{*}\right)$ :

$$
E^{*}=E\left(\gamma^{*}\right)=\frac{c^{2} \sqrt{\frac{a}{\delta}}}{a+\beta \sqrt{\frac{a}{\delta}}+\delta\left(\sqrt{\frac{a}{\delta}}\right)^{2}}=\frac{c^{2}}{2 \sqrt{a \delta}+\beta}
$$

By exploiting (12), $E^{*}$ can also be expressed as follows:

$$
\begin{aligned}
E^{*} & =\frac{c^{2}}{2 \sqrt{a d(a d+c b)}+2 a d+c b} \\
& =\frac{c(\sqrt{a d+c b}-\sqrt{a d})}{b(\sqrt{a d+c b}+\sqrt{a d})}=\frac{c\left(\sqrt{1+\frac{c b}{a d}}-1\right)}{b\left(\sqrt{1+\frac{c b}{a d}}+1\right)}
\end{aligned}
$$

Moreover, by using (4) one can easily prove that $E^{*}$ in (13) is always less than 1, that is: $E^{*} \leq 1$. Fig. 3 shows an example, where the efficiency of a linear system $\mathbf{H}(s)$ characterized by parameters $a=0.2, b=0.6, c=0.8, d=0.1$ and $P_{\max }=100 \mathrm{~W}$ is reported on the output plane $\left(y_{2}, u_{2}\right)$. The values of $\gamma^{*}$ and $E^{*}$ are: $\gamma^{*}=2$ and $E^{*}=0.8889$.

Property 3: The efficiency $E(t)$ of a linear system is constant along straight lines exiting from the origin of the input plane $\left(y_{1}, u_{1}\right)$ :

$$
u_{1}=\alpha y_{1}, \quad \alpha \in[0, \infty]
$$

and reaches its maximum value $E^{*}$ for:

$$
\alpha=\sqrt{\frac{d}{a(a d+b c)}}=\alpha^{*}
$$




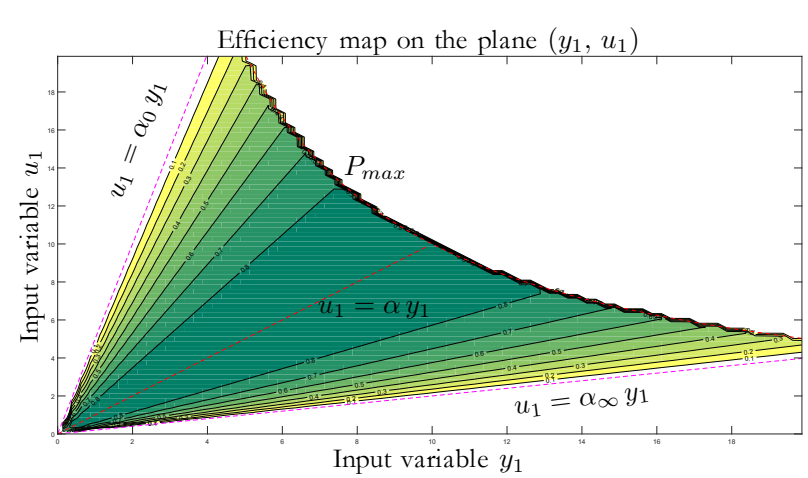

Fig. 4. Efficiency map shown on the plane of the input variables $\left(y_{1}, u_{1}\right)$.

Proof. By substituting (14) in (8), it is possible to express $E(t)$ as a function of parameter $\alpha$ :

$$
E(t)=\frac{(1-a \alpha)[(a d+b c) \alpha-d]}{b^{2} \alpha}=E(\alpha)
$$

Efficiency $E(\alpha)$ in (16) can also be rewritten as follows:

$$
E(\alpha)=\frac{-d+\beta \alpha-\eta \alpha^{2}}{b^{2} \alpha} \text { where }\left\{\begin{array}{l}
\beta=2 a d+c b \\
\eta=a(a d+b c)
\end{array}\right.
$$

By deriving $E(\alpha)$ with respect to $\alpha$, one obtains:

$$
\frac{\partial E(\alpha)}{\partial \alpha}=\frac{\left(d-\eta \alpha^{2}\right) b^{2}}{\left(b^{2} \alpha\right)^{2}}
$$

Efficiency $E(\alpha)$ is maximized when $\frac{\partial E(\alpha)}{\partial \alpha}=0$, that is when $\alpha=\sqrt{\frac{d}{\eta}}$. By using $\eta$ from (17), one directly obtains relation (15).

By means of (16), the maximum efficiency $E^{*}=E\left(\alpha^{*}\right)$ is:

$$
E^{*}=E\left(\alpha^{*}\right)=\frac{-d+\beta \sqrt{\frac{d}{\eta}}-\eta\left(\sqrt{\frac{d}{\eta}}\right)^{2}}{b^{2} \sqrt{\frac{d}{\eta}}}=\frac{\beta-2 \sqrt{\eta d}}{b^{2}}
$$

By using (17), $E^{*}=E\left(\alpha^{*}\right)$ can also be expressed as:

$$
\begin{aligned}
E^{*} & =\frac{2 a d+c b-2 \sqrt{a d(a d+c b)}}{b^{2}} \\
& =\frac{c(\sqrt{a d+c b}-\sqrt{a d})}{b(\sqrt{a d+c b}+\sqrt{a d})}=\frac{c\left(\sqrt{1+\frac{c b}{a d}}-1\right)}{b\left(\sqrt{1+\frac{c b}{a d}}+1\right)}
\end{aligned}
$$

Obviously, the maximum efficiency value $E^{*}$ obtained in (18) coincides with the maximum efficiency value $E^{*}$ obtained in (13). Moreover, slopes $\alpha$ and $\gamma$ are related as follows:

$$
\alpha=\frac{d \gamma+1}{(a d+b c) \gamma+a}, \quad \gamma=\frac{a \alpha-1}{d-\alpha(a d+b c)} .
$$

Fig. 4 shows the efficiency of the same linear system $\mathbf{H}(s)$ considered in Fig. 3 reported on the input plane $\left(y_{1}, u_{1}\right)$. In this case, it is: $\alpha^{*}=1$ and $E^{*}=0.8889$.

\section{EFFICIENCY OF NONLINEAR SYSTEMS}

Two different cases will be considered: A) a linear system $\mathbf{H}(s)$ with additional nonlinear terms; B) a full nonlinear system $\dot{\mathbf{x}}=\mathbf{f}(\mathbf{x}, \mathbf{u})$.

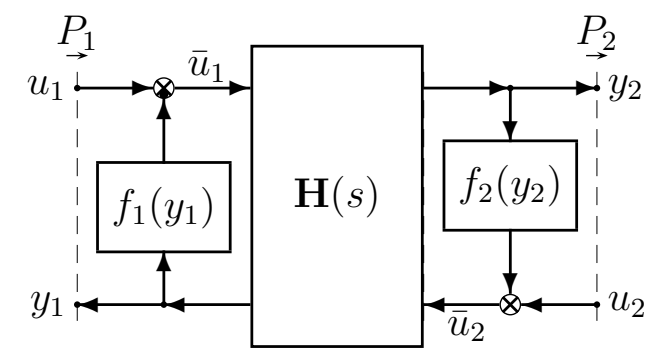

Fig. 5. Linear system $\mathbf{H}(s)$ with additional nonlinear friction terms.

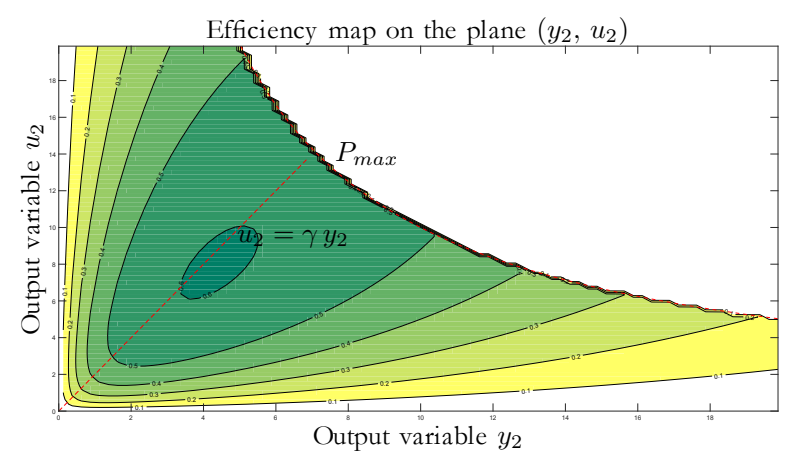

Fig. 6. Efficiency map on plane $\left(y_{2}, u_{2}\right)$ in presence of nonlinearities.

\section{A. Linear system $\mathbf{H}(s)$ with additional nonlinear terms}

The block scheme shown in Fig. 5 describes a linear system $\mathbf{H}(s)$ with additional nonlinear friction terms. The structure of nonlinear functions $f_{1}\left(y_{1}\right)$ and $f_{2}\left(y_{2}\right)$ is supposed to be the following:

$f_{1}\left(y_{1}\right)=b_{c 1} \operatorname{sgn}\left(y_{1}\right)+b_{d 1} y_{1}^{2}, \quad f_{2}\left(y_{2}\right)=b_{c 2} \operatorname{sgn}\left(y_{2}\right)+b_{d 2} y_{2}^{2}$.

The efficiency of the overall nonlinear system, having $\left(y_{1}, u_{1}\right)$ and $\left(y_{2}, u_{2}\right)$ as input and output power sections, can be expressed as follows:

1) Efficiency on the output plane $\left(y_{2}, u_{2}\right)$ :

$$
E\left(y_{2}, u_{2}\right)=\frac{u_{2} y_{2}}{u_{1} y_{1}}=\frac{u_{2} y_{2}}{\left(\bar{u}_{1}+f_{1}\left(y_{1}\right)\right) y_{1}}
$$

where

$$
\left[\begin{array}{l}
\bar{u}_{1} \\
y_{1}
\end{array}\right]=\left[\begin{array}{cc}
\frac{d}{c} & \frac{1}{c} \\
\frac{a d+b c}{c} & \frac{a}{c}
\end{array}\right]\left[\begin{array}{l}
\bar{u}_{2} \\
y_{2}
\end{array}\right], \quad \bar{u}_{2}=u_{2}+f_{2}\left(y_{2}\right) .
$$

2) Efficiency on the input plane $\left(y_{1}, u_{1}\right)$ :

$$
E\left(y_{1}, u_{1}\right)=\frac{u_{2} y_{2}}{u_{1} y_{1}}=\frac{\left(\bar{u}_{2}-f_{2}\left(y_{2}\right)\right) y_{2}}{u_{1} y_{1}}
$$

where

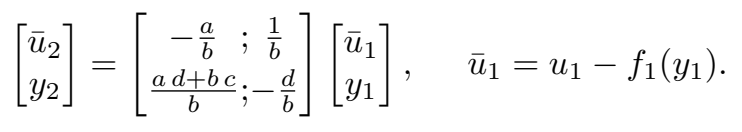

By referring to the linear system $\mathbf{H}(s)$ defined in Sec. II$\mathrm{B}$ and introducing nonlinear friction coefficients $b_{c 1}=0.6$, $b_{c 2}=0.9, b_{d 1}=0.02$ and $b_{d 2}=0.03$, one obtains the efficiency maps on planes $\left(y_{2}, u_{2}\right)$ and $\left(y_{1}, u_{1}\right)$ shown in Fig. 6 and Fig. 7, respectively. 


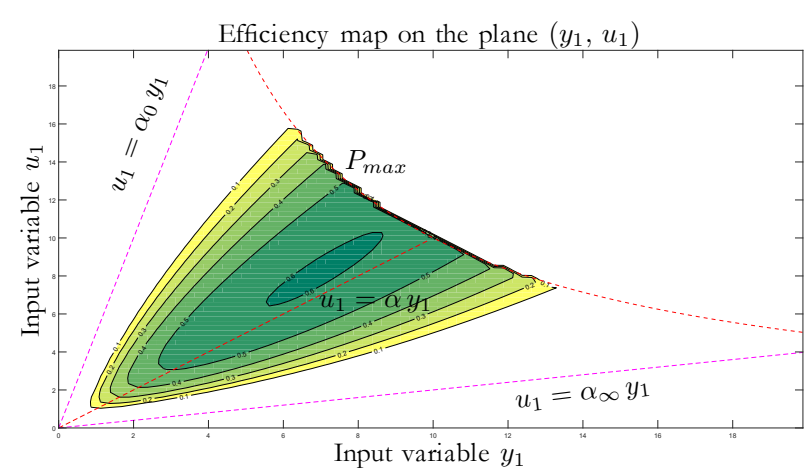

Fig. 7. Efficiency map on plane $\left(y_{1}, u_{1}\right)$ in presence of nonlinearities.

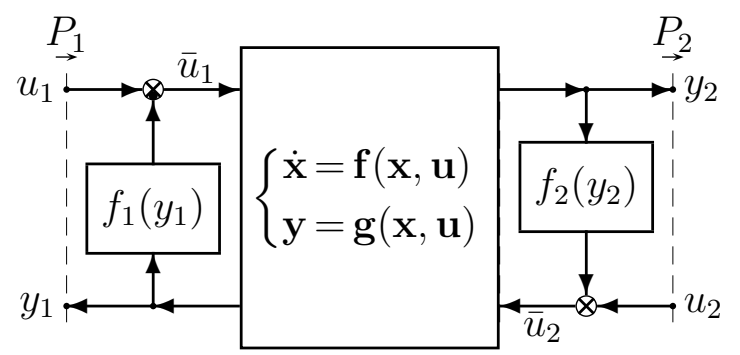

Fig. 8. Nonlinear system $\dot{\mathbf{x}}=\mathbf{f}(\mathbf{x}, \mathbf{u})$ with nonlinear friction terms.

\section{B. A full nonlinear system $\dot{\mathbf{x}}=\mathbf{f}(\mathbf{x}, \mathbf{u})$.}

The block scheme shown in Fig. 8 describes a nonlinear system in presence of additional nonlinear friction terms. In steady-state condition, the considered nonlinear system is described by the following nonlinear static equations:

$$
\left\{\begin{aligned}
\mathbf{0} & =\mathbf{f}\left(\mathbf{x}_{s s}, \bar{u}_{1}, \bar{u}_{2}\right) \\
{\left[\begin{array}{c}
y_{1} \\
y_{2}
\end{array}\right] } & =\mathbf{g}\left(\mathbf{x}_{s s}, \bar{u}_{1}, \bar{u}_{2}\right)
\end{aligned}\right.
$$

The first equation $0=\mathbf{f}\left(\mathbf{x}_{s s}, \bar{u}_{1}, \bar{u}_{2}\right)$ must be solved with respect to the internal state space vector $\mathbf{x}_{s s}=\mathbf{f}^{-1}\left(\bar{u}_{1}, \bar{u}_{2}\right)$. By using $\mathbf{x}_{s s}$, the output nonlinear equation of system (21) can be expressed as follows:

$$
\left[\begin{array}{l}
y_{1} \\
y_{2}
\end{array}\right]=\mathbf{g}\left(\mathbf{f}^{-1}\left(\bar{u}_{1}, \bar{u}_{2}\right), \bar{u}_{1}, \bar{u}_{2}\right) \text {. }
$$

This system consists of two nonlinear static equations which are function of the four power variables $\bar{u}_{1}, y_{1}, \bar{u}_{2}$ and $y_{2}$. By solving this nonlinear system with respect to $\left(\bar{u}_{1}, y_{1}\right)$ and $\left(\bar{u}_{2}, y_{2}\right)$ one obtains, respectively, the following solutions:

$$
\left[\begin{array}{l}
\bar{u}_{1} \\
y_{1}
\end{array}\right]=\left[\begin{array}{l}
p_{1}\left(\bar{u}_{2}, y_{2}\right) \\
q_{1}\left(\bar{u}_{2}, y_{2}\right)
\end{array}\right], \quad\left[\begin{array}{l}
\bar{u}_{2} \\
y_{2}
\end{array}\right]=\left[\begin{array}{l}
p_{2}\left(\bar{u}_{1}, y_{1}\right) \\
q_{2}\left(\bar{u}_{1}, y_{1}\right)
\end{array}\right] .
$$

The efficiency of the overall nonlinear system can be expressed in the following ways:

1) Efficiency on the output plane $\left(y_{2}, u_{2}\right)$ :

$$
E\left(y_{2}, u_{2}\right)=\frac{u_{2} y_{2}}{u_{1} y_{1}}=\frac{u_{2} y_{2}}{\left(\bar{u}_{1}+f_{1}\left(y_{1}\right)\right) y_{1}}
$$

where $\bar{u}_{1}=p_{1}\left(\bar{u}_{2}, y_{2}\right), y_{1}=q_{1}\left(\bar{u}_{2}, y_{2}\right)$ and $\bar{u}_{2}=u_{2}+f_{2}\left(y_{2}\right)$. Functions $p_{1}\left(\bar{u}_{2}, y_{2}\right)$ and $q_{1}\left(\bar{u}_{2}, y_{2}\right)$ are defined in (22).
2) Efficiency on the input plane $\left(y_{1}, u_{1}\right)$ :

$$
E\left(y_{1}, u_{1}\right)=\frac{u_{2} y_{2}}{u_{1} y_{1}}=\frac{\left(\bar{u}_{2}-f_{2}\left(y_{2}\right)\right) y_{2}}{u_{1} y_{1}}
$$

where $\bar{u}_{2}=p_{2}\left(\bar{u}_{1}, y_{1}\right), y_{2}=q_{2}\left(\bar{u}_{1}, y_{1}\right)$ and $\bar{u}_{1}=u_{1}-f_{1}\left(y_{1}\right)$. Functions $p_{2}\left(\bar{u}_{1}, y_{1}\right)$ and $q_{2}\left(\bar{u}_{1}, y_{1}\right)$ are defined in (22).

\section{Simulation RESUlTS}

\section{A. Efficiency of an electric DC motor.}

Consider the POG block scheme of an electric DC motor shown in Fig. 9. The parameters of the system have the following meaning: $L$ is the stator inductance, $R$ is the stator resistance, $J$ is the rotor moment of inertia, $B$ is the rotor linear friction coefficient and $K$ is the torque constant of motor. The POG state space equations of the system are the

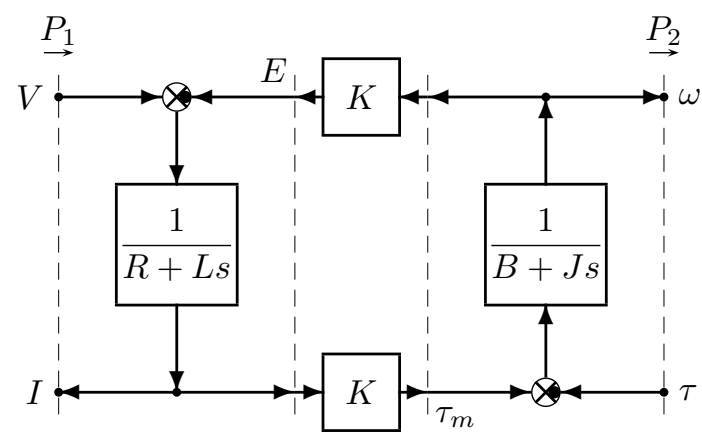

Fig. 9. POG block scheme of an electric DC motor.

following:

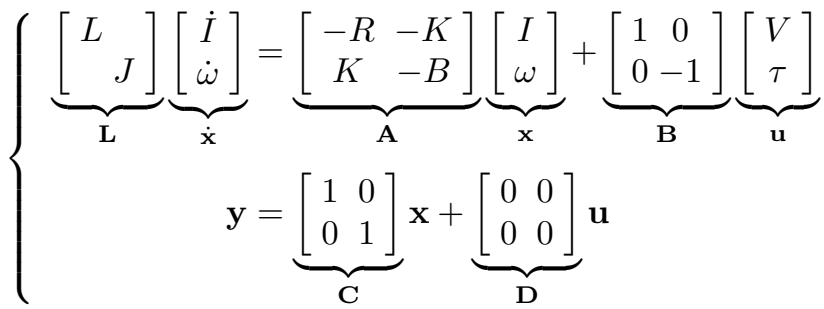

Matrix $\mathbf{H}_{0}$, for the considered electric DC motor, is:

$$
\mathbf{H}_{0}=\left[\begin{array}{cc}
\frac{B}{R B+K^{2}} & \frac{K}{R B+K^{2}} \\
\frac{K}{R B+K^{2}} & \frac{-R}{R B+K^{2}}
\end{array}\right]=\left[\begin{array}{cc}
a & b \\
c & -d
\end{array}\right]
$$

Parameters $a, b, c, d$ defined in (2) are the following:

$a=\frac{B}{R B+K^{2}}, \quad b=\frac{K}{R B+K^{2}}=c, \quad d=\frac{R}{R B+K^{2}}$.

In this case, the maximum efficiency $E^{*}$, see (18), is:

$E^{*}=\frac{\left(\sqrt{1+\frac{K^{2}}{R B}}-1\right)}{\left(\sqrt{1+\frac{K^{2}}{R B}}+1\right)}=\frac{(\sqrt{1+q}-1)}{(\sqrt{1+q}+1)}$ where $q=\frac{K^{2}}{R B}$.

Efficiency $E^{*}(q)$ of the electric DC motor as a function of parameter $q$ is shown in Fig. 10. From the figure, it is evident 


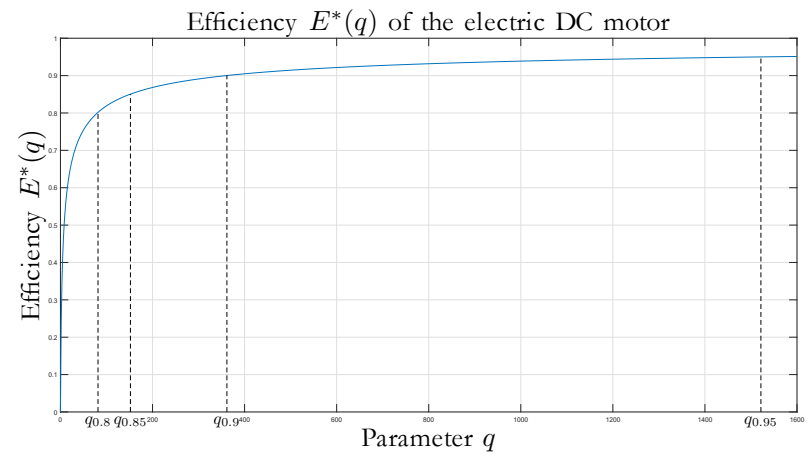

Fig. 10. DC motor efficiency $E^{*}(q)$ as a functions of parameter $q$.

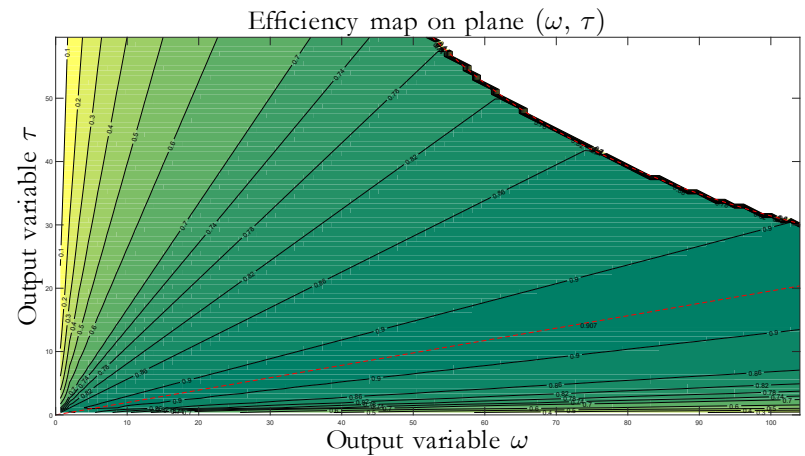

Fig. 11. Efficiency map of the DC motor on the output plane $(\omega, \tau)$.

that:

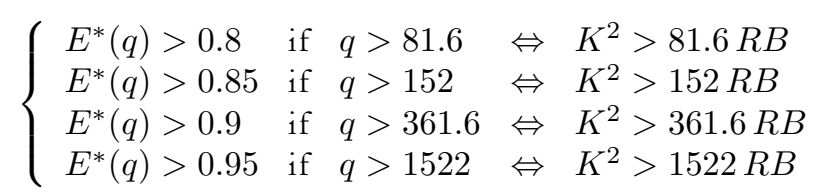

From (10), it is known that on the plane of the output variables $(\omega, \tau)$, the maximum efficiency $E^{*}(q)$ is obtained for the following slope:

$$
\gamma^{*}=\sqrt{\frac{B\left(R B+K^{2}\right)}{R}}=B \sqrt{1+\frac{K^{2}}{R B}}=B \sqrt{1+q} .
$$

Consequently, for constant $q$ (i.e. constant maximum efficiency $E^{*}$ ), the optimal slope $\gamma^{*}$ is proportional to coefficient $B$ : DC motors characterized by a large value of $B$ are suitable for working in high torque-low speed operating regions, whereas DC motors characterized by a low value of $B$ are suitable for working in low torque-high speed operating regions. The efficiency map of the DC motor on the plane of the output variables $(\omega, \tau)$ is shown in Fig. 11.

\section{B. Efficiency of a mechanical gear transmission.}

Let us consider the POG block scheme shown in Fig. 12 describing the dynamics of a mechanical gear transmission system. The parameters of the system have the following meaning: $J_{1}, b_{1}$ and $R_{1}$ are the moment of inertia, the linear friction coefficient and the radius of the first gear, respectively. Equivalently, $J_{2}, b_{2}$ and $R_{2}$ are the moment of inertia, the linear friction coefficient and the radius of the second gear, respectively. Finally, $K$ is the stiffness of the spring acting in between the two gears. The state space

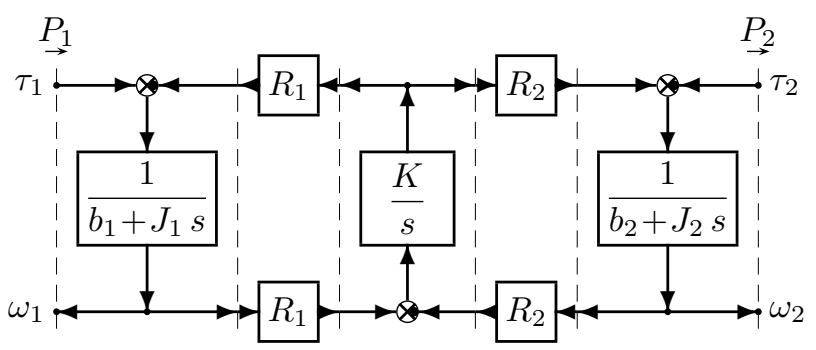

Fig. 12. POG block scheme of a mechanical gear transmission system.

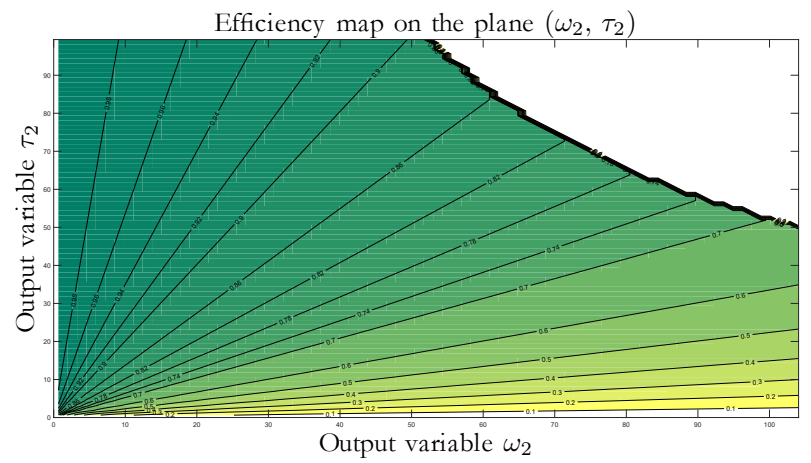

Fig. 13. Efficiency map of the gear transmission on output plane $\left(\omega_{2}, \tau_{2}\right)$.

equations of the considered gear transmission system are the following:

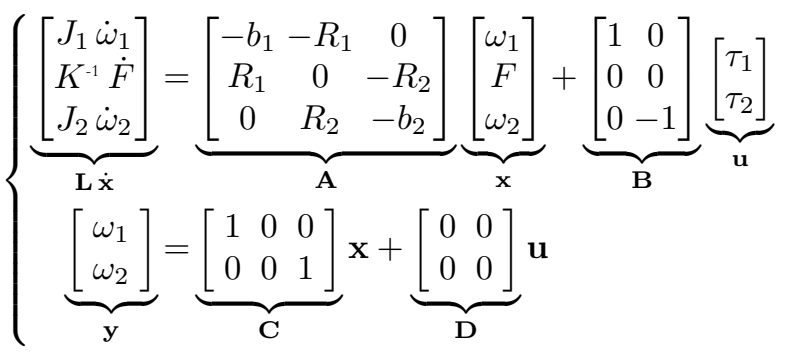

Matrix $\mathbf{H}_{0}$ of the considered system, see (1) and (2), is:

$$
\mathbf{H}_{0}=\left[\begin{array}{cc}
\frac{1}{b_{1}+b_{2} r^{2}} & \frac{-r}{b_{1}+b_{2} r^{2}} \\
\frac{r}{b_{1}+b_{2} r^{2}} & \frac{-r^{2}}{b_{1}+b_{2} r^{2}}
\end{array}\right]=\left[\begin{array}{cc}
a & b \\
c & -d
\end{array}\right]
$$

where $r=\frac{R_{1}}{R_{2}}$. For the gear transmission system, see (18), the maximum efficiency $E^{*}$ is equal to 1 :

$$
E^{*}=\frac{c\left(\sqrt{1+\frac{c b}{a d}}-1\right)}{b\left(\sqrt{1+\frac{c b}{a d}}+1\right)}=\frac{-(\sqrt{1-1}-1)}{(\sqrt{1-1}+1)}=1
$$

On the plane of the output variables $\left(\omega_{2}, \tau_{2}\right)$, the maximum efficiency $E^{*}=1$ is obtained for $\gamma^{*}=\infty$ when $\omega=0$ :

$$
\gamma^{*}=\sqrt{\frac{a}{d(a d+c b)}}=\sqrt{\frac{\left(b_{1}+b_{2} r^{2}\right)^{2}}{r^{2}\left(r^{2}-r^{2}\right)}}=\infty .
$$

The efficiency map of the gear transmission system on the output plane $\left(\omega_{2}, \tau_{2}\right)$ is shown in Fig. 13. When $K \rightarrow \infty$, the dynamic equation of the reduced system is:

$$
\left(J_{1}+r^{2} J_{2}\right) \dot{\omega}_{1}=-\left(b_{1}+r^{2} b_{2}\right) \omega_{1}+[1-r] \mathbf{u}
$$


One can easily verify that matrix $\mathbf{H}_{0}$ of the reduced system (25) is exactly equal to the one obtained in (24) for the original system (23), meaning that the efficiency of a mechanical gear transmission does not depend on the value of the dynamic element $K$. This result agrees with what is stated in Prop. 1.

\section{Efficiency of a electric PMSM.}

The steady-state equations of a Permanent Magnet Synchronous Motor (PMSM) in the rotating frame $\Sigma_{\omega}$, see [4], have the following structure:

$$
\underbrace{\left[\begin{array}{cc|c}
p R_{s} & -p^{2} \omega_{m} L_{s e} & 0 \\
p^{2} \omega_{m} L_{s e} & p R_{s} & \omega^{\omega} K_{q} \\
\hline 0 & -{ }^{\omega} K_{q} & b_{m}
\end{array}\right]\left[\begin{array}{c}
{ }^{\omega} I_{d} \\
{ }^{\omega} I_{q} \\
\hline \omega_{m}
\end{array}\right]-\left[\begin{array}{c}
{ }^{\omega} V_{d} \\
{ }^{\omega} V_{q} \\
\hline-\tau_{e}
\end{array}\right]=\left[\begin{array}{c}
0 \\
0 \\
\frac{0}{0}
\end{array}\right]}_{\mathbf{f}\left(\mathbf{x}_{s s}, \mathbf{u}\right)=\mathbf{0}}
$$

The meaning of the system parameters is reported in Tab. I.

\begin{tabular}{|c|l|}
\hline$p$ & Number of polar expansions; \\
\hline$R_{s}$ & Stator phase resistance $(p=1) ;$ \\
\hline$L_{s e}$ & $L_{s}+\frac{1}{2} M_{s 0} ;$ \\
\hline$L_{s}$ & Stator phase self inductance coefficient $(p=1) ;$ \\
\hline$M_{s 0}$ & Maximum value of mutual inductance between stator phases; \\
\hline$b_{m}$ & Rotor linear friction coefficient; \\
\hline$\omega_{m}$ & Motor angular velocity; \\
\hline${ }^{\omega} K_{q}$ & Motor torque constant; \\
\hline${ }^{\omega} V_{d}$ & Direct component of the motor input voltage vector; \\
\hline${ }^{\omega} V_{q}$ & In-quadrature component of the motor input voltage vector; \\
\hline${ }^{\omega} I_{d}$ & Direct component of the motor output current vector; \\
\hline${ }^{\omega} I_{q}$ & In-quadrature component of the motor output current vector; \\
\hline$\tau_{e}$ & External load torque applied to the motor; \\
\hline
\end{tabular}

\section{TABLE I}

PARAMETERS OF SySTEM (26).

1) Controlled PMSM: if voltage ${ }^{\omega} V_{d}$ is chosen in order to impose the condition ${ }^{\omega} I_{d}=0$, the nonlinear static equations (26) simplify as follows:

$$
\left[\begin{array}{c}
{ }^{\omega} V_{d} \\
{ }^{\omega} V_{q} \\
\hline-\tau_{e}
\end{array}\right]=\left[\begin{array}{c|c}
-p^{2} \omega_{m} L_{s e} & 0 \\
p R_{s} & { }^{\omega} K_{q} \\
\hline-{ }^{\omega} K_{q} & b_{m}
\end{array}\right]\left[\begin{array}{c}
{ }^{\omega} I_{q} \\
\hline \omega_{m}
\end{array}\right]
$$

By solving with respect to ${ }^{\omega} I_{q},{ }^{\omega} V_{d}$ and ${ }^{\omega} V_{q}$, one obtains:

$$
\left[\begin{array}{c}
{ }^{\omega} I_{q} \\
{ }^{\omega} V_{d} \\
{ }^{\omega} V_{q}
\end{array}\right]=\left[\begin{array}{c}
\frac{b_{m} \omega_{m}+\tau_{e}}{\omega K_{q}} \\
-\frac{p^{2} \omega_{m} L_{s e}\left(b_{m} \omega_{m}+\tau_{e}\right)}{\omega K_{q}} \\
\frac{\omega_{m}{ }^{\omega} K_{q}^{2}+R_{s} \tau_{e} p+R_{s} b_{m} p \omega_{m}}{\omega K_{q}}
\end{array}\right]
$$

Without the first equation, system (27) can be rewritten as:

$$
\left[\begin{array}{l}
u_{1} \\
u_{2}
\end{array}\right]=\underbrace{\left[\begin{array}{c}
{ }^{\omega} V_{q} \\
\tau_{e}
\end{array}\right]}_{\mathbf{u}}=\left[\begin{array}{cc}
p R_{s} & { }^{\omega} K_{q} \\
{ }^{\omega} K_{q} & -b_{m}
\end{array}\right] \underbrace{\left[\begin{array}{c}
{ }^{\omega} I_{q} \\
\omega_{m}
\end{array}\right]}_{\mathbf{y}}
$$

By solving with respect to $\mathbf{y}$, it results:

$$
\left[\begin{array}{l}
y_{1} \\
y_{2}
\end{array}\right]=\underbrace{\left[\begin{array}{c}
\omega \\
I_{q} \\
\omega_{m}
\end{array}\right]}_{\mathbf{y}}=\underbrace{\left[\begin{array}{cc}
\frac{b_{m}}{p R_{s} b_{m}+{ }^{\omega} K_{q}^{2}} & \frac{{ }^{\omega} K_{q}}{p R_{s} b_{m}+{ }^{\omega} K_{q}^{2}} \\
\frac{\omega K_{q}}{p R_{s} b_{m}+{ }^{\omega} K_{q}^{2}}-\frac{p R_{s}}{p R_{s} b_{m}+{ }^{\omega} K_{q}^{2}}
\end{array}\right]}_{\mathbf{H}_{0}} \underbrace{\left[\begin{array}{c}
{ }^{\omega} V_{q} \\
\tau_{e}
\end{array}\right]}_{\mathbf{u}}
$$

The obtained matrix $\mathbf{H}_{0}$ has the structure defined in (2), where parameters $a, b, c, d$ are:

$$
a=\frac{b_{m}}{\Delta}, \quad b=\frac{{ }^{\omega} K_{q}}{\Delta}=c, \quad d=\frac{p R_{s}}{\Delta}
$$

where $\Delta=p R_{s} b_{m}+{ }^{\omega} K_{q}^{2}$. For the considered PMSM, the maximum efficiency value $E^{*}$, see (18), is:

$$
E^{*}=\frac{(\sqrt{1+q}-1)}{(\sqrt{1+q}+1)} \quad \text { where } \quad q=\frac{{ }^{\omega} K_{q}^{2}}{p R_{s} b_{m}} .
$$

2) Electric PMSM: the general case: if the electric motor is not controlled, both currents ${ }^{\omega} I_{d}$ and ${ }^{\omega} I_{q}$ are different from zero. In this case, system (26) can be rewritten as follows:

$$
\underbrace{\left[\begin{array}{c}
0 \\
{ }^{\omega} K_{q} \omega_{m} \\
b_{m} \omega_{m}+\tau_{e}
\end{array}\right]}_{\mathbf{b}}=\underbrace{\left[\begin{array}{cccc}
-p R_{s} & p^{2} \omega_{m} L_{s e} & 1 & 0 \\
-p^{2} \omega_{m} L_{s e} & -p R_{s} & 0 & 1 \\
0 & { }^{\omega} K_{q} & 0 & 0
\end{array}\right]}_{\mathbf{A}} \underbrace{\left[\begin{array}{c}
\omega \\
{ }^{\omega} I_{d} \\
I_{q} \\
{ }^{\omega} V_{d} \\
\omega^{\omega} V_{q}
\end{array}\right]}_{\mathbf{x}}
$$

The solution of system (31) is:

$$
\mathbf{x}=\underbrace{\left[\begin{array}{c}
\frac{p \omega_{m} L_{s e}\left(b_{m} \omega_{m}+\tau_{e}\right)}{\omega K_{q} R_{s}} \\
\frac{b_{m} \omega_{m}+\tau_{e}}{\omega} K_{q} \\
0 \\
\frac{p\left(p^{2} \omega_{m}^{2} L_{s e}^{2}+R_{s}^{2}\right)\left(b_{m} \omega_{m}+\tau_{e}\right)+{ }^{\omega} K_{q}^{2} R_{s} \omega_{m}}{\omega K_{q} R_{s}}
\end{array}\right]}_{\mathbf{x}_{0}}+\underbrace{\left[\begin{array}{c}
\alpha \\
\alpha p^{2} \omega_{m} L_{s e}
\end{array}\right]}_{\operatorname{ker}(\mathbf{A})}
$$

where $\alpha \in \mathbf{R}$ is an arbitrary real parameter. Solution (32) can also be expressed as follows:

$$
\mathbf{x}=\left[\begin{array}{c}
I_{d 0}+\alpha \\
I_{q 0} \\
\alpha p R_{s} \\
V_{q 0}+\alpha p^{2} \omega_{m} L_{s e}
\end{array}\right] \quad \text { where } \quad\left[\begin{array}{c}
I_{d 0} \\
I_{q 0} \\
0 \\
V_{q 0}
\end{array}\right]=\mathbf{x}_{0} .
$$

The power $P_{1}={ }^{\omega} V_{d}{ }^{\omega} I_{d}+{ }^{\omega} V_{q}{ }^{\omega} I_{q}$ entering the system is given by:

$$
P_{1}=\alpha p R_{s}\left(I_{d 0}+\alpha\right)+I_{q 0}\left(V_{q 0}+\alpha p^{2} \omega_{m} L_{s e}\right) .
$$

The efficiency $E\left(\omega_{m}, \tau_{e}\right)=\frac{P_{2}}{P_{1}}$ of the PMSM on the output plane can be expressed as follows:

$$
E\left(\omega_{m}, \tau_{e}\right)=\frac{\omega_{m} \tau_{e}}{\alpha p R_{s}\left(I_{d 0}+\alpha\right)+I_{q 0}\left(V_{q 0}+\alpha p^{2} \omega_{m} L_{s e}\right)}
$$

where $P_{2}=\omega_{m} \tau_{e}$ is the power exiting the system. Efficiency $E\left(\omega_{m}, \tau_{e}\right)$ is a function of parameter $\alpha$. The maximum efficiency is obtained when the partial derivative of function $E\left(\omega_{m}, \tau_{e}\right)$ with respect to $\alpha$ is equal to zero:

$$
\frac{\partial E\left(\omega_{m}, \tau_{e}\right)}{\partial \alpha}=\frac{-\omega_{m} \tau_{e}\left(p R_{s}\left(I_{d 0}+2 \alpha\right)+I_{q 0} p^{2} \omega_{m} L_{s e}\right)}{\left(p R_{s}\left(\alpha I_{d 0}+\alpha^{2}\right)+I_{q 0}\left(V_{q 0}+\alpha p^{2} \omega_{m} L_{s e}\right)\right)^{2}}=0
$$


By solving with respect to $\alpha$, one obtains:

$$
\alpha^{*}=\frac{-R_{s} I_{d 0}-I_{q 0} p \omega_{m} L_{s e}}{2 R_{s}} .
$$

By substituting (32) in (35), it results:

$$
\alpha^{*}=-\frac{p \omega_{m} L_{s e}\left(b_{m} \omega_{m}+\tau_{e}\right)}{{ }^{\omega} K_{q} R_{s}}=-I_{d 0}
$$

By substituting (36) in (33), one obtains:

$$
\mathbf{x}^{*}=\left[\begin{array}{c}
0 \\
\frac{b_{m} \omega_{m}+\tau_{e}}{\omega} K_{q} \\
-\frac{p^{2} \omega_{m} L_{s e}\left(b_{m} \omega_{m}+\tau_{e}\right)}{\omega} K_{q} \\
\frac{\omega_{m}{ }^{\omega} K_{q}^{2}+R_{s} \tau_{e} p+R_{s} b_{m} p \omega_{m}}{{ }^{\omega} K_{q}}
\end{array}\right]
$$

Note that the obtained solution $\mathbf{x}^{*}$ is equal to the solution obtained in (28) when the control strategy imposes the condition ${ }^{\omega} I_{d}=0$.

\section{Electric PMSM: parameters identification.}

The analysis presented in the previous sections can be used, for example, to estimate the parameters of a PMSM. The efficiency map of electric PMSM is typically given on the output plane $\left(y_{2}, u_{2}\right)=\left(\omega_{m}, \tau_{e}\right)$. For these systems, Eq. (20) can be rewritten as follows:

$$
\left[\begin{array}{c}
\bar{u}_{1} \\
{ }^{\omega} I_{q}
\end{array}\right]=\left[\begin{array}{cc}
\frac{d}{c} & \frac{1}{c} \\
\frac{a d+b c}{c} & \frac{a}{c}
\end{array}\right]\left[\begin{array}{c}
\bar{u}_{2} \\
\omega_{m}
\end{array}\right]
$$

where $a, b, c, d$ are the coefficients of matrix $\mathbf{H}_{0}$ defined in (29) and inputs $u_{1}$ and $u_{2}$ are defined as follows:

$$
\left\{\begin{array}{l}
u_{2}=\tau_{e}=\bar{u}_{2}-b_{c} \operatorname{sign}\left(\omega_{m}\right) \\
u_{1}={ }^{\omega} V_{q}=\bar{u}_{1}+R_{s q}{ }^{\omega} I_{q}^{2}
\end{array}\right.
$$

Parameters $b_{c}$ and $R_{s q}$ in (38) are, respectively, the amplitude of the Coulomb friction acting on the rotor, and the dissipative coefficient accounting for the Joule losses of the motor. The efficiency of the PMSM depends on the values of the dissipative parameters $b_{m}, b_{c}, R_{s}, R_{s q}$, which are usually unknown. Nevertheless, their values can be estimated if the efficiency map of the system on the output plane $\left(\omega_{m}, \tau_{e}\right)$ in given. Let us consider, for example, the normalized efficiency map of an industrial three-phase synchronous electric motor shown in Fig. 14. From this figure, one can read the efficiency of the system on a properly chosen set of points. Then, a least square algorithm can be used to minimize the mean squared error between the real efficiency on the selected points and the efficiency of the PMSM model on the same points. By applying this procedure to the normalized efficiency map shown in Fig. 14, one obtains the following estimated parameters for the considered industrial PMSM: $b_{m}=6.4610^{-3}[\mathrm{Nm} /(\mathrm{rad} / \mathrm{s})], b_{c}=1.97$ $[\mathrm{Nm}], R_{s}=2.8[\mathrm{~m} \Omega], R_{s q}=3.52\left[\mu V / A^{2}\right]$. Fig. 15 shows the obtained estimated efficiency maps of the considered industrial electric motor. Comparing Fig. 15 with Fig. 14 one can see a very good matching between the estimated

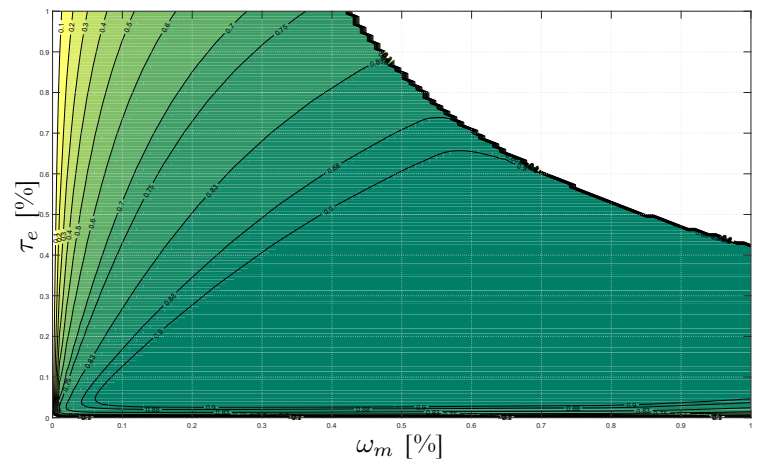

Fig. 14. Normalized efficiency map of an industrial electric motor on plane $\left(\omega_{m}, \tau_{e}\right)$.

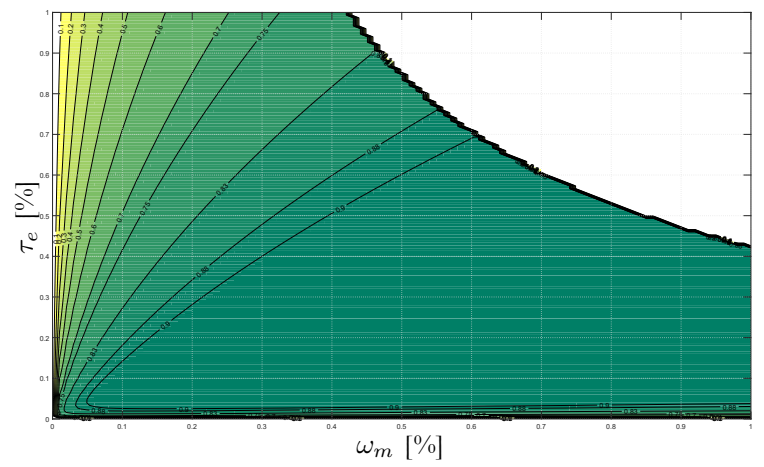

Fig. 15. Estimated efficiency map of an industrial electric motor on plane $\left(\omega_{m}, \tau_{e}\right)$.

and the actual efficiency maps, denoting the effectiveness of the presented procedure.

\section{CONCLUSiOnS}

In this paper, a theoretical analysis regarding the power flow-based efficiency of linear and nonlinear systems has been presented. This analysis highlights the main relations affecting the efficiency of the analyzed physical system and shows how to change the system parameters in order to enlarge the high-efficiency region as much as possible. Based on this analysis and using a least square algorithm, a procedure allowing to estimate the parameters of a PMSM has been presented and its effectiveness has been verified by applying it to an actual PMSM.

\section{REFERENCES}

[1] Quntao An, Li Sun, "On-line Parameter Identification for Vector Controlled PMSM Drives Using Adaptive Algorithm”, IEEE Vehicle Power and Propulsion Conference (VPPC), Harbin, China, September 3-5, 2008.

[2] Xiaoliang Jiang, Pindong Sun, Z. Q. Zhu, "Modeling and Simulation of Parameters Identification for PMSM Based on EKF", International Conference on Computer, Mechatronics, Control and Electronic Engineering (CMCE), Changchun, China, August 24-26, 2010.

[3] R. Zanasi, "The Power-Oriented Graphs Technique: System modeling and basic properties", IEEE Vehicle Power and Propulsion Conference, Lille, France, September 1-3, 2010.

[4] M. Fei, R. Zanasi, F. Grossi, "Modeling of Multi-phase Permanent Magnet Synchronous Motors under Open-phase Fault Condition”, IEEE International Conference on Control and Automation (ICCA), Santiago, Chile, December 19-21, 2011. 